\title{
Design and validation of a simple eye-tracking system
}

\author{
Dorion B. Liston ${ }^{1,2^{*}}$ \\ ${ }^{1}$ NASA Ames Research Center \\ Sol Simpson ${ }^{3}$ \\ Lily R. Wong ${ }^{1,2}$ \\ Mark Rich ${ }^{4}$ \\ Leland S. Stone ${ }^{1}$ \\ ${ }^{2}$ San José State University $\quad{ }^{3}$ iSolverSolutions \\ ${ }^{4}$ New York University
}

\begin{abstract}
To address the need for portable systems to collect high-quality eye movement data for field studies, this paper shows how one might design, test, and validate the spatiotemporal fidelity of a homebrewed eye-tracking system. To assess spatial and temporal precision, we describe three validation tests that quantify the spatial resolution and temporal synchronization of data acquisition. First, because measurement of pursuit eye movements requires a visual motion display, we measured the timing of luminance transitions of several candidate LCD monitors so as to ensure sufficient stimulus fidelity. Second, we measured eye position as human observers $(n=20)$ ran a nine-point calibration in a clinical-grade chin rest, delivering eye-position noise of $0.22 \mathrm{deg}$ (range: 0.09-0.29 deg) and accuracy of $0.97 \mathrm{deg}$ (range: $0.54-1.89 \mathrm{deg}$ ). Third, we measured the overall processing delay in the system to be $5.6 \mathrm{~ms}$, accounted for by the response dynamics of our monitor and the duration of one camera frame. The validation methods presented can be used: 1) to ensure that eye-position accuracy and precision are sufficient to support scientific and clinical studies and are not limited by the hardware or software, and 2) the eyetracker, display, and experiment-control software are effectively synchronized.
\end{abstract}

CR Categories: • Hardware Functional verification • Humancentered computing Empirical studies in $\mathrm{HCI}$

Keywords: oculometric, eye position, tracking precision

\section{Introduction}

Pioneering clinical oculomotor work investigated the signs of psychiatric illness [Diefendorf and Dodge 1908] using a falling photographic plate to record a time series of horizontal eye movements. In the intervening century, various inherently non-algorithmic mechanical, electrical, or optical eye-movement recording techniques have been introduced, including: skin-mounted electrodes [Marg 1951], a cornea-mounted suction cup mechanically attached to a stylus [Yarbus 1967], electromagnetic tracking using search coils [Robinson 1963], and a purpose-built electro-optical device to generate continuous electrical signals from Purkinje images [Cornsweet and Crane 1973]. Over the past two decades, videobased techniques have become the standard non-invasive eyetracking method in humans. For some time, special-purpose hardware has been necessary to generate a system with sufficiently high temporal and spatial resolution to be adequate for scientific or clin-

\footnotetext{
*email: dliston@arc.nasa.gov

(C) 2016 Association for Computing Machinery. ACM acknowledges that this contribution was authored or co-authored by an employee, contractor or affiliate of the United States government. As such, the United States Government retains a nonexclusive, royalty-free right to publish or reproduce this article, or to allow others to do so, for Government purposes only. ETRA '16, March 14-17, 2016, Charleston, SC, USA (C) 2016 ACM. ISBN 978-1-4503-4125-7/16/03 ...\$15.00 DOI: http://dx.doi.org/10.1145/2857491.2857534
}

ical eye-tracking applications. Recent developments in PC interfaces, camera technology, and open-source software have made it possible to match the performance of proprietary commercial systems with relatively inexpensive off-the-shelf components, for some tracking applications. We describe a design process for a home-made, high-speed, high-resolution eye-tracking system using inexpensive commercially-available hardware and straightforward signal-processing software that relies on a simple 2D pupil-thresholding algorithm. We also show how to validate the overall system's spatial accuracy and precision as well as the temporal synchronization of the eye-position data with the stimulus display.

\section{Design}

The overall design goal was to build a non-invasive eye-tracking system that can collect high spatio-temporal precision 2D eye-position data from inexperienced human subjects in non-laboratory settings, using relatively inexpensive commercial-off-the-shelf components. The electronic components are a high-speed camera, a display, and a PC, with associated ergonomic components, including: a chinrest, subject chair, and table as integral parts of the system. The system was designed in a modular fashion to allow for straightforward substitution of hardware (camera, display) and software (data acquisition package) components, for maximum flexibility, with a multi-core PC powerful enough to run our eyetracker in tandem with an experiment-control software package (e.g., MATLAB, PsychoPy). To minimize cost and maximize flexibility, we programmed the software for our system using opensource packages (SDL, OpenGL, OpenCV, Python).

The ergonomic components were also designed to optimize the quality of eye-position data, while still allowing for a deployable device that could be used by naïve subjects in non-laboratory settings (e.g., a field hospital). The chinrest (UHCO, Houston, TX) clamps to the tabletop and had adjustable chin and forehead rests to minimize head-movement artifacts while maintaining subject comfort. The center of the display was positioned ten degrees above the height of the subject's eye to prevent pupil occlusion by the upper eyelid given that eyelid opens fully with upward gaze [Stoller and Meyer 1994]. The subject chair was a medical-grade ergonomic stool (Soma Ergonomics, Ceres, CA) with sufficient travel to position the subject's eye at a fixed height regardless of torso size. To create a sturdy, yet collapsible, table base, we used 2"x2" extruded aluminum to build a 3D rectangular base (20" deep x 40" wide x 36 " high) that collapses (4" deep x 40" wide x 36 " high) for transport. As both the camera and the chinrest are mechanically attached to the tabletop, movement from the subject (or the floor) will also vibrate the camera to some extent; to dampen these vibrations, we used as heavy a tabletop as was practical for one person to carry (42" x $22.5 "$ x 1.5 " hardwood, $40 \mathrm{lbs})$.

\subsection{Optical components: camera, lens, lighting}

There are a number of potential camera configurations (e.g., sensor, lens, lighting, etc.) that could deliver the needed level of spatial precision (noise $<1 / 4 \mathrm{deg}$ ) at high frame rates $(>250 \mathrm{~Hz}$ ). We focused on the most widely-available, flexible, and relatively inex- 
pensive options. To narrow the field of cameras, we limited the interface to USB3 (as opposed to PCIe, Thunderbolt, or Ethernet) and the sensor type to monochrome CMOS (as opposed to CCD). Our chosen camera (XIMEA, Golden, $\mathrm{CO}$ ) was positioned under the monitor at a distance of $18.5 "(470 \mathrm{~mm})$ from the subject, requiring a lens able to focus at that distance while projecting an eye image of $\sim 512 \times 512$ pixels on the $2 / 3$ " sensor with resolution of $2048 \times 1088$. The relatively large sensor affords the flexibility to image one or both eyes at high resolution. We restricted our search to fixed focal length c-mount lenses with working distances of 470 $\mathrm{mm}$ or less, ultimately using a $75 \mathrm{~mm}$ macro lens (Computar, Tokyo, Japan).

We chose a 48-LED $850-\mathrm{nm}$ illuminator $(12 \mathrm{~V}, 500 \mathrm{~mA})$, positioned alongside the camera and offset to allow for dark-pupil tracking. At the working distance of 18 in., the measured NIR power density at the position of the subject's cornea is $\sim 0.3$ $\mathrm{mW} / \mathrm{cm}^{2}$ (Ophir Orion laser power meter, NIR head), providing ample illumination for eye-tracking at $250 \mathrm{~Hz}$, yet well below the safety limit of $10 \mathrm{~mW} / \mathrm{cm}^{2}$ common to several standards, including: (1) OSHA 1910.97 (for periods of more than 6 minutes), (2) "Threshold limit values for chemical substances and physical agents, and Biological exposure indices," published by the American Conference of Governmental Industrial Hygienists (1995), and (3) European Standard EN 62471 "Photobiological safety of lamps and lamp systems".

\subsection{Stimulus display}

Motion perception depends critically on the quality of sampled motion [Watson 2013], as does the smooth pursuit eye-movement response [Churchland and Lisberger 2000; Liston and Stone 2014]. As our system was designed to provide a smooth motion signal to elicit smooth pursuit movements, we limited our search to LCD displays with HD-level resolution (1920x1080) that support a refresh rate at the current standard for high-performance gaming (144 $\mathrm{Hz})$. Although LCD displays have some disadvantages: luminance depends on viewing angle, luminance signals in different locations interact, and sampled motion is held for the entire frame rather than pulsed [Wang and Nikolic 2011], the fixed head position afforded by our chinrest and the simplicity of the high-contrast spot commonly used in clinical or experimental oculomotor tasks discounted these disadvantages, especially given the low cost, availability, and steady development in high-performance LCD displays.

The percept of a stimulus moving on a sampled display is related to a number of display and motion-sampling properties, including: sample-and-hold vs. sample-and-blank motion, spatial resolution, pixel response time, and display refresh rate. Together, these factors influence the physical sequencing of the retinal image that provides the initial drive for smooth pursuit eye movements and contributes to the percept of motion that sustains the pursuit movement during steady-state tracking. Following methods described in detail previously [Becker 2008; Watson 2010], we measured the timing of luminance transitions of the BenQ XL2420Z $144 \mathrm{~Hz}$ monitor using a test pattern to capture a time series record of luminance values at a central point on the monitor with a high-speed camera (Ximea, Golden, $\mathrm{CO}$ ). We reversed the contrast polarity of the grating every 30 frames at $144 \mathrm{~Hz}$, and recorded luminance samples at 1 $\mathrm{kHz}$. To examine high-contrast transitions, we alternated between the maximum 8-bit grayscale gun values of 0 and 255 . To estimate the delay $(\mu)$ associated with the leading and trailing edges of a moving stimulus, we fit a cumulative Gaussian function [Watson 2010] to the black-to-white and white-to-black luminance transitions, synchronized to the graphics card buffer-flip, as shown in Figure 1.
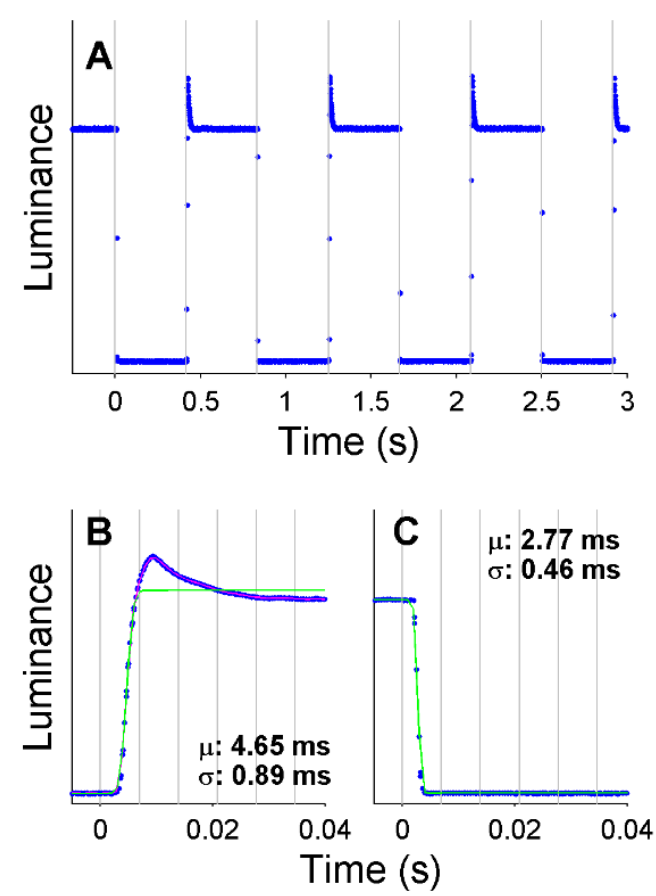

Figure 1: Timing of luminance transitions. Panel A plots a time series record of luminance changes at one point in the center of the monitor with a high-contrast, temporally-modulated, square-wave test pattern. Filled blue circles show the luminance measurements. The filled blue circles in panel B shows the time course of the transition from black to white, with several transitions superimposed. Panel C shows the corresponding transition from white to black. Following Watson [2010], we fit the shape of this luminance change with the integral of a Gaussian shape (solid green line).

\subsection{Signal Processing}

The PC hardware is the most flexible component in the system; it need only be sufficiently powerful to run the eyetracker and the experiment-control software simultaneously (ASUS P8Z77-i motherboard, Intel i7 $3770 \mathrm{CPU}, 3.5 \mathrm{GHz}, 2 \times 4 \mathrm{~GB}$ Corsair DDR3 RAM).

We developed a simple, modular, tracking program that allows for straightforward substitution of the camera hardware, image processing algorithm, and visualization of the results of the image processing. We used a standard algorithm to find the pupil centroid by applying a single grayscale threshold and flood-filling to eliminate non-contiguous pixels. We used this simple algorithm to maximize speed and minimize the possibility of unknown biases arising from a more complicated algorithm. The simple-threshold algorithm, however, fails ungracefully if the image is poorly composed for eye-tracking (e.g., drooping eyelid, eyelash shadows), if illumination is inadequate, or if the threshold is set incorrectly. The pupilthresholding algorithm is associated with three data structures: a set of image processing parameters (e.g., a grayscale threshold used to identify pupil pixels), a set of intermediate variables (e.g., various binary images of contiguous and non-contiguous sub-threshold pixels), and a set of measurements (i.e., hor. position, ver. position, and area) returned by the algorithm sent via network broadcast.

We used PsychoPy [Peirce 2007], an open-source Python-based experiment-control program, to display stimuli and control stimulus timing. We synchronized the eye-position signals sent from our eyetracker with the stimulus using calls to a single high-resolution timer. We used the ioHub utility distributed with PsychoPy to receive the UDP broadcast from the eyetracker and save hdf5 data. 
We report two tests of our eye-tracking system: first, eye-position accuracy and precision are quantified using the fixation variability during a calibration run; and second, the system timing delays between the display and eyetracker is measured by turning the eyetracking camera toward the display and using it to track a moving spot on the display instead of an eye.

\subsection{Spatial eye-position noise}

To quantify the internal processing noise of our eyetracker, we used a set of glass eyes (green, hazel, and blue) ranging in size from 12$30 \mathrm{~mm}$ (Van Dyke's Taxidermy Supply, Granite Quarry, NC). To distinguish biological noise from system noise, we collected a ninepoint $( \pm 15 \mathrm{deg})$ calibration from a small sample $(\mathrm{n}=20)$ of human observers, and compared those to control data measured with the glass eyes to assess data quality [Holmqvist et al. 2012; Reingold 2014]. For each of the nine calibration points, we recorded 1 second of data at $250 \mathrm{~Hz}$.

A six-parameter fit was used to convert pupil position in pixels to degrees of visual angle [Beutter and Stone 1998]. For our sample of 20 human observers, our eyetracker delivered an average eye position noise of $0.22 \mathrm{deg}$ (range: $0.09-0.29 \mathrm{deg}$ ), defined as the standard deviation of eye position during a fixation, and an average accuracy of $0.97 \mathrm{deg}$ (range: $0.54-1.89 \mathrm{deg}$ ), defined as the RMS deviation from a perfect calibration averaged across the nine calibration points. These levels are not dissimilar to fixation precisions of $0.32 \mathrm{deg}$ measured from a research-grade eye-tracking system with subjects using a chinrest [Liston and Stone 2014]. When using a chinrest instead of a bite bar, the accuracy and precision of these measures are both degraded by small head translations between and during recorded fixations [Young and Sheena 1975]. From first principles, noise in measured eye position could arise from a number of factors which cluster into two categories: (1) system noise,
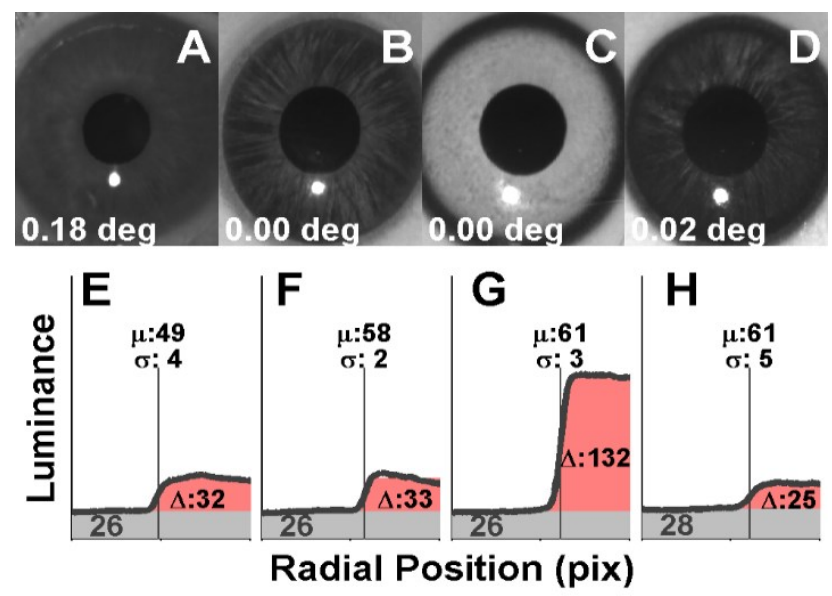

Figure 2: Comparison of human and glass eye luminance profiles. Panels A-D show images of a human eye (A) and three glass eyes $(B-D)$ of similar size, all recorded with our eyetracker under similar illumination and camera conditions. The inset text gives the eye-position precision during a nine-point calibration. We also computed the mean radial luminance profile of each image (shown in panels $E$-H) using the centroid computation, and fit them with a cumulative Gaussian shape. Pixels containing the corneal reflection of the IR illuminator were discarded before taking the radial average. Note that two of the glass-eye radial profiles ( $F$ and $H$ ) are similar to that of a human eye (E). including luminance fluctuations, sensor noise, or camera movement; or (2) biological noise, including head movement artifacts, microsaccades, ocular drift, or tremor. To determine the contribution of system noise to our measured precision, we collected calibration data from three realistic glass eyes (Figure 2B-D) similar in size to a human eye (Figure 2A), but with different patterns of iris coloration, yielding slightly different luminance profiles when averaged radially around the pupil centroid (Figure 2E-H).

We then used the calibration parameters from our sample of human observers [Beutter and Stone 1998] to measure the position noise of glass eyes during "fixation", $0.01 \mathrm{deg}$ on average (range: 0.00 to $0.03 \mathrm{deg}$ ), our best estimate of the internal system noise. These noise levels were substantially lower than values measured from any human observer, consistent with the idea that biological noise from small-amplitude eye movements (e.g., microsaccades, drift, tremor), or head movement artifacts contribute substantially to human eye-position noise.

\subsection{Temporal synchronization}

To validate the dynamics of the LCD display and the synchronization with our eye-tracking software, we tracked the center of a black spot moving sinusoidally at $1 \mathrm{kHz}$ (using a 512x128 pixel image), as shown in Figure 3. The overall delay in our stimulus command and eye-tracking loop includes three steps. First, the experimentcontrol program records a buffer flip on the graphics card, timestamped with a high-resolution timer, which sends an updated image to the display. Second, the updated image is displayed on the monitor, limited by the dynamics of white-to-black and black-towhite luminance transitions (Figure 1). Third, the camera sensor transduces the image for the exposure duration of a single frame (1 $\mathrm{ms}$ at $1 \mathrm{kHz}$ ) and streams it to our tracking program, where it is time-stamped with the same high-resolution timer. Because both the graphics-card buffer flip and the acquisition of the camera image by our tracking program were time-stamped by the same timer, any delay associated with computing the pupil centroid or sending the resulting data structure back to the experiment-control program is effectively outside this closed loop. We measured the overall delay to be $5.6 \mathrm{~ms}$, which can be accounted for by the $4.65 \mathrm{~ms}$ delay in the black-to-white transitions for the BenQ XL2420Z and the 1 $\mathrm{ms}$ exposure duration of each image. We conclude from this test that our experiment-control program, stimulus display, and eyetracker are synchronized to within the temporal resolution of our camera and display hardware.

\section{Discussion}

We designed and tested a simple eye-tracking system to demonstrate that one can build and validate a homebrewed system to collect high-precision 2D eye-position data from human subjects in experimental or clinical settings. The total cost of electronic components in this system designed to elicit and measure smooth pursuit eye movements was approximately $\$ 3500$. We measured the eye-position noise to be $\sim 0.2$ deg during fixation, consistent with previous values recorded from subjects on a chinrest [Liston and Stone 2014]. Indeed, the measured internal system noise was minimal $(<0.01 \mathrm{deg})$, indicating that additional gains in precision from the eyetracker for this application are pointless. We found that movements (e.g., eye-and-head translation, ocular drift, tremor, microsaccades) made by human observers dominate our eye-position uncertainty while fixating, consistent with many previous observations [Yarbus 1967]. Of these movements, microsaccades have amplitudes of $\sim 0.25 \mathrm{deg}$, matching our noise level, but were not commonly found in our eye-position records. Drift has an amplitude of $<0.08 \mathrm{deg}(<5 \mathrm{~min})$, and tremor has an amplitude of $<0.02$ 
$\operatorname{deg}(<1 \mathrm{~min}$ at $80 \mathrm{~Hz})$ [Martinez-Conde et al. 2004], both too low to account for noise levels of $0.2 \mathrm{deg}$. This leaves eye-and-head translations during fixation as the most likely limiting factor, shifting the focus to the mechanical and ergonomic properties of the system to minimize head movements on the chinrest and camera vibration. Last, we measured the overall delay in the processing loop containing the stimulus display, eyetracker, and data transmission, and found that the total delay was only $5.6 \mathrm{~ms}$, well-matched to the response dynamics of the LCD display $(4.6 \mathrm{~ms})$ and the sampling interval for measuring luminance transitions ( $1 \mathrm{~ms})$. We conclude that by using the design principles and validation methods described above, one can build a relatively inexpensive, custombuilt system that can deliver the high level of spatiotemporal fidelity needed for scientific or clinical research.

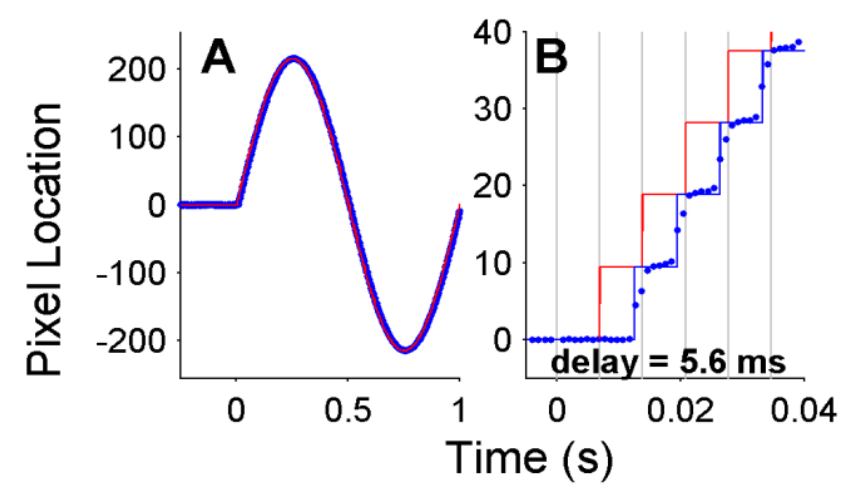

Figure 3: Tracking a moving spot. Although nearly-superimposed at this scale, panel A plots the position of the black spot as sent to the graphics card as a solid red line (i.e., discrete position steps at $144 \mathrm{~Hz}$ ) while the filled blue circles show the measured position (in pixels) returned by our eye-tracking software. Panel B plots the first four position steps as the spot starts moving. We estimated a delay of $5.6 \mathrm{~ms}$ between the position of the spot as given by the graphics card (solid red line) and its measured positon on the monitor (filled blue circles) with a 1-parameter fit (solid blue line).

\section{Acknowledgements}

We thank the Office of Naval Research Force Health Protection program (N0001415IP00028/30) for funding. We are grateful to Brent Beutter, Jeff Mulligan and Don Kalar for helpful comments on this manuscript, and to Chris Collacott for technical assistance.

\section{References}

AMERICAN CONFERENCE OF GOVERNMENTAL INDUSTRIAL HyGIENISTS. 1995. Threshold Limit Values for Chemical Substances and Physical Agents and Biological Exposure Indices. American Conference of Governmental Industrial Hygienists.

BECKER, M.E. 2008. Motion-blur evaluation: A comparison of approaches. Journal of the SID 16, 989-1000.

BeutTer, B.R. AND STONE, L.S. 1998. Human motion perception and smooth eye movements show similar directional biases for elongated apertures. Vision Res 38, 1273-1286.

Churchland, M.M. And Lisberger, S.G. 2000. Apparent motion produces multiple deficits in visually guided smooth pursuit eye movements of monkeys. J Neurophysiol 84 , 216-235.

CORnsweEt, T.N. AND CRANE, H.D. 1973. Accurate twodimensional eye tracker using first and fourth Purkinje images. J Opt Soc Am 63, 921-928.

DiEFENDORF, A.R. AND DodGe, R. 1908. An experimental study of the ocular reactions of the insane from photographic records. Brain 31, 451-489.

Holmovist, K., Nystrom, M. AND Mulvey, F. 2012. Eye tracker data quality: What it is and how to measure it. In Proceedings of the Symposium on Eye Tracking Research and Applications, New York: ACM, 45-52.

INTERNATIONAL ELECTROTECHNICAL COMMISSION. 2006. International Standard IEC 62471:2006/CIE S 009:2002. In Photobiological safety of lamps and lamp systems.

Liston, D.B. AND StOne, L.S. 2014. Oculometric assessment of dynamic visual processing. $J$ Vis $14,12$.

MARG, E. 1951. Development of electro-oculography; standing potential of the eye in registration of eye movement. AMA Arch Ophthalmol 45, 169-185.

Martinez-Conde, S., MackniK, S.L. And Hubel, D.H. 2004. The role of fixational eye movements in visual perception. Nat Rev Neurosci 5, 229-240.

OCCUPATIONAL SAFETY AND HEALTH ADMINISTRATION. Occupational Safety and Health Standards. In Nonionizing radiation, Standard number 1910.97.

Peirce, J.W. 2007. PsychoPy--Psychophysics software in Python. J Neurosci Methods 162, 8-13.

REINGOLD, E.M. 2014. Eye tracking research and technology: Towards objective measurement of data quality. Visual Cognition 22, 635-652.

RoBINSON, D.A. 1963. A method of measuring eye movement using a scleral search coil in a magnetic field. IEEE Trans Biomed Eng 10, 137-145.

StOller, S.H. AND MeYer, D.R. 1994. Quantitating the change in upper eyelid position during downgaze. Ophthalmology 101, 1604-1607.

WANG, P. AND NikOLIC, D. 2011. An LCD monitor with sufficiently precise timing for research in vision. Front Hum Neurosci 5, 85.

WATson, A.B. 2010. Display motion blur: Comparison of measurement methods. Journal of the SID 18, 179-190.

WAtson, A.B. 2013. High frame rates and human vision: A view through the window of visibility. SMPTE Motion Imaging Journal 122, 18-32.

YARBUS, A.L. 1967. Eye movements and vision. Plenum Press, New York.

YounG, L.R. AND SHEENA, D. 1975. Eye-movement measurement techniques. Am Psychol 30, 315-330. 